\title{
An Image Processing Algorithm to Detect Exudates in Fundus Images
}

\author{
Anisa Anjum \\ Department of Electronics and \\ Communication, Islamiah Institute \\ of Technology, National Park \\ Road, Bannerghatta Road, \\ Bengaluru, Karnataka, India, \\ anisaanjum830@gmail.com
}

\author{
B Mehataj \\ Department of Electronics and \\ Communication, Islamiah Institute \\ of Technology, National Park \\ Road, Bannerghatta Road, \\ Bengaluru, Karnataka, India, \\ mehtajbasha96@gmail.com
}

\author{
Shaik Mubeena \\ Department of Electronics and \\ Communication, Islamiah Institute \\ of Technology, National Park \\ Road, Bannerghatta Road, \\ Bengaluru, Karnataka, India, \\ ssheeba888@gmail.com
}

\author{
Sudharani Astagi \\ Department of Electronics and \\ Communication, Islamiah Institute of \\ Technology, National Park Road, \\ Bannerghatta Road, Bengaluru, \\ Karnataka, India, \\ sudharaniastagi@gmail.com
}

\section{Senthil Lekha}

Head of Department \& Associate Professor, Department of Electronics and Communication, Islamiah Institute of Technology, National Park Road, Bannerghatta Road, Bengaluru, Karnataka, India

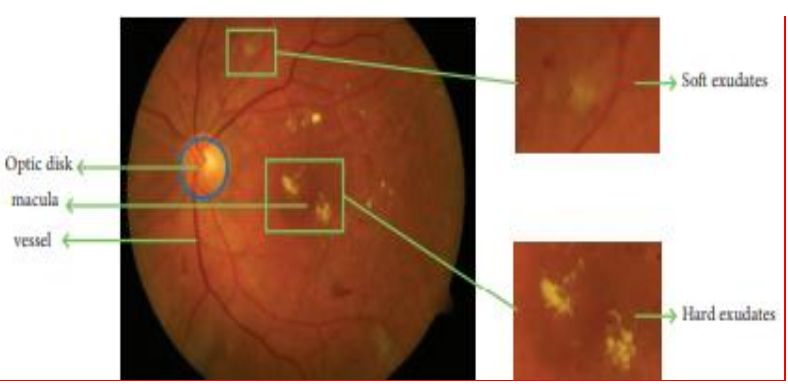

Fig 1. Example of retinal fundus image with exudates regions.

\section{A. Hard Exudates}

They are yellowish-white in color and presents in and around the macula region [2].

\section{B. Soft Exudates}

A cotton wool spot, also known as soft exudates, are faded white in color and often lie below blood vessels [2].

This paper concentrates on detecting these exudates using automated algorithm that employs image processing and statistics.

\section{LITERATURE SURVEY}

The paper [3] proposes a computer assisted diagnosis based on digital processing of Retinal Images. The Retinal images are used to detect DR in advance. An initial image processing stage isolates blood vessel, Microaneurysms, the optic-disc, and hard exudates in order to extract the featured used by the SVM. However, the drawback of this work is the optic disk detection is carried out using blood vessel extraction. This can result in erroneous results, as 
blood vessels can be abruptly placed in the retina. And the SVM process used is highly time consuming.

According to [4], DR detection method was able to detect exudates, blood vessel and hemorrhages with high accuracy. The high accuracy of classification results showed that the proposed system is reliable, and the implementation of the proposed system could tackle the limitation of the manual screening process. However, for some cases, where the images were out of focus, the algorithm fails to detect some of the DR features. Drawback of this work is the optic disk detection is carried out using blood vessel extraction. This can result in erroneous results, as blood vessels can be abruptly placed in the retina. This methodology provides accuracy of only $85 \%$.

The authors in [5] propose a method that can be used for detection of diabetic retinopathy by analyzing presence of exudates in fundus images of eyes efficiently. Algorithm could mask optic disc and non-exudates like blood vessels and clots. The algorithm is computationally less expensive, and complexity is also less and has achieved a high efficiency of $87 \%$ accuracy. However, few more texture features for extracting exudates also features may be added to achieve at most accuracy.

\section{PROPOSED ALGORITHM}

The flow followed for the algorithm is shown in Fig. 2. The fundus images are fed to the video processor. The video processor houses image processing algorithm detect the exudates. The algorithm has the concepts of Image acquisition. The fundus image will select the suitable color models, to which exudates can be clearly distinguished from the background (Performing segmentation to extract exudates) Unfortunately, the texture and color of exudates match with optic disk and along with exudates, optic disk also gets extracted. Thus, removal of optic disk is performed using morphologically noise removal techniques. Once the exudates are retrieved, the area of spread is estimated. This area parameter has to be fed to CBIR (Content Based Image Retrieval) to retrieve images which have same parameters.

\section{A. Image Acquisition}

We acquired sixty images each with different volume of exudates and different appearance, one of them shown in Fig. 3.

\section{B. Color Processing}

The default color model is RGB, where R is Red, $\mathrm{G}$ is Green \& B is Blue. Each color value varies from 0 to 255 , which requires 8 bits for its representation. If all the 3 colors are combined together, one has to work with $8 \times 3=24$ bits/pixel, which might be expensive with respect to memory \& time. Thus, working on individual colors turns out to be feasible. Based on the color of background and exudates the green model is chosen, as shown in Fig. 4.

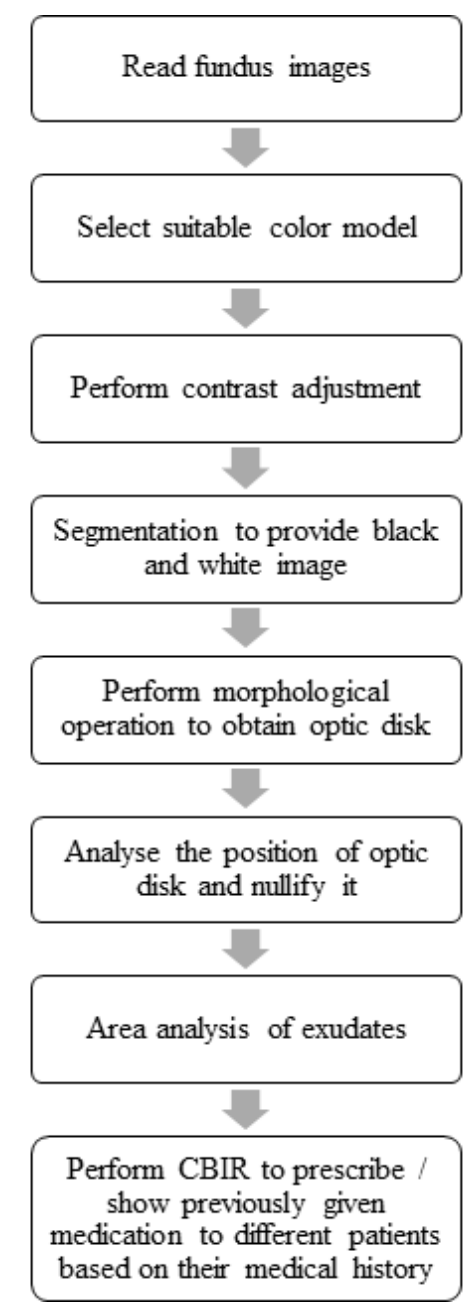

Fig 2. Flowchart of the algorithm

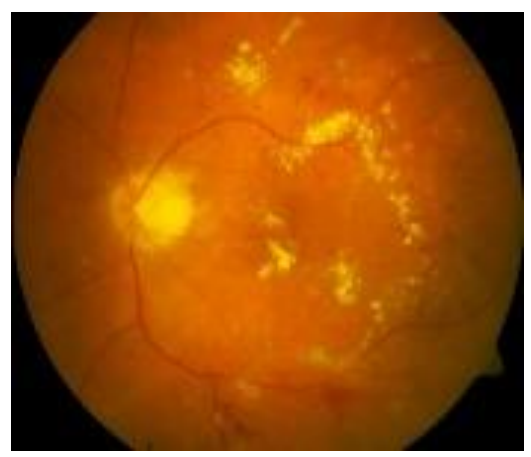

Fig 3. Retinal image

\section{Contrast Adjustment}

Contrast adjustment remaps image intensity values to the full display range of the data type, such that the bottom $1 \%$ and the top $1 \%$ of all pixel values are saturated [6], as shown in Fig. 5. 


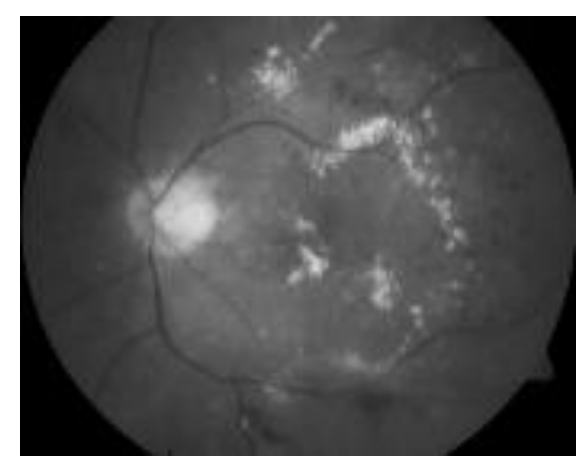

Fig 4. Green image

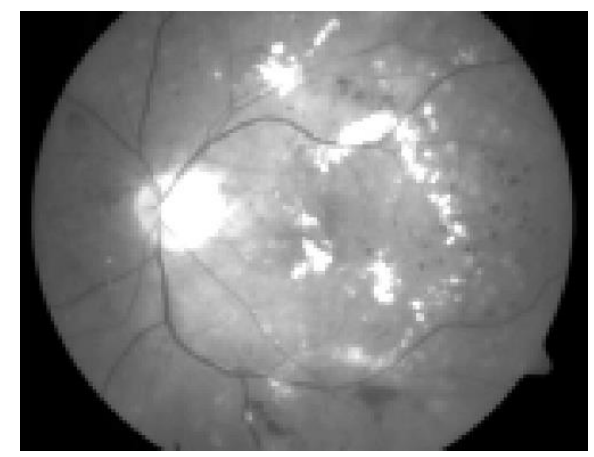

Fig 5. Contrast Adjusted Image

\section{Segmented Image}

To track objects of interact easily, the color image is converted to white and black image. By finding minimum and maximum to get mean of the exudates for the images then by calculating histogram value of maximum values where the exudates lay and also coding to get most suitable curve analysis formula. Once threshold is obtained segmentation is performed using following logic, where ca is contrast adjusted image.

$$
\operatorname{seg}_{r, c}= \begin{cases}\text { white, } & \text { if } c a_{r, c} \geq \text { threshold } \\ \text { black, } & \text { if } c a_{r, c} \leq \text { threshold }\end{cases}
$$

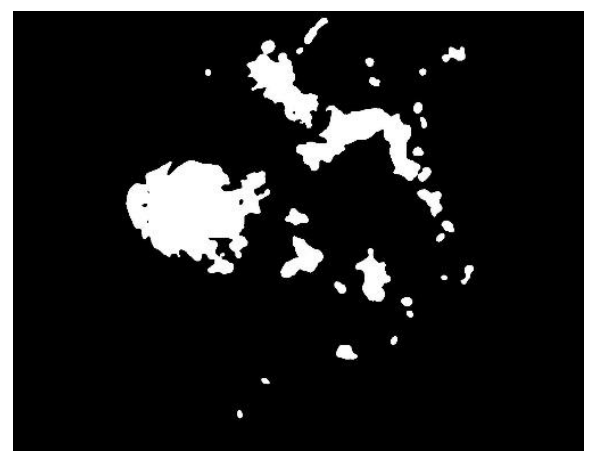

Fig 6. Segmented image

\section{E. Morphological operations:}

Morphological operations are used to either add or remove extra pixels to/from the borders of objects in an image, based on the neighboring pixels [7]. According to
[8], the most basic morphological operations are dilation and erosion. Dilation expands the boundaries of white objects in the image, while erosion reduces them. The image after performing erosion and dilation is shown in Fig. 7.

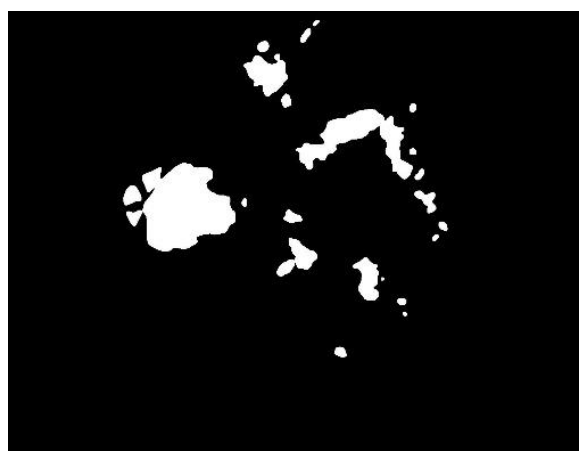

Fig 7. Noise removed image

The noise removed image is divided into different blobs and the spread of each blob is found out, the one with highest pixel count is declared as optic disk and is removed, thus retaining only the exudates, as shown in Fig. 8.

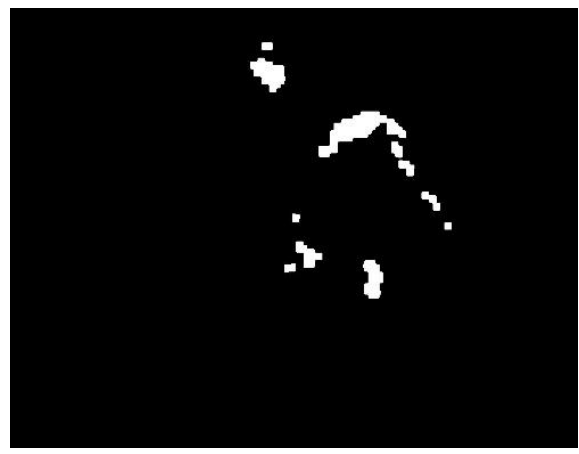

Fig 8. Images with exudates extracted

\section{RESULTS}

The algorithm has been tested on 60 images, and worked well on 54 images. The accuracy is calculated to be $90 \%$.

\section{CONCLUSION}

In this paper, an efficient framework for early detection of Diabetic Retinopathy has been developed. The optic disk is tracked and eliminated. The experiment shows that the proposed DR detection method was able to detect exudates with high accuracy. Thus, using our project, we can detect exudates among diabetic retinopathy patients and help the needy. As its fully automated process there is no need of human interference.

\section{REFERENCES}

[1] Long, Shengchun, et al. "Automatic detection of hard exudates in color retinal images using dynamic threshold and SVM classification: algorithm development and evaluation." BioMed research international 2019 (2019). 
[2] Prakash, N. B., and D. Selvathi. "An efficient approach for detecting exudates in diabetic retinopathy images." (2016).

[3] E. V. Carrera, A. González and R. Carrera, "Automated detection of diabetic retinopathy using SVM," 2017 IEEE XXIV International Conference on Electronics, Electrical Engineering and Computing (INTERCON), Cusco, 2017, pp. 1-4, doi: 10.1109/INTERCON.2017.8079692.

[4] Z. A. Omar, M. Hanafi, S. Mashohor, N. F. M. Mahfudz and M. Muna'im, "Automatic diabetic retinopathy detection and classification system," 2017 7th IEEE International Conference on System Engineering and Technology (ICSET), Shah Alam, 2017, pp. 162-166, doi: 10.1109/ICSEngT.2017.8123439.

[5] P. Patil, P. Shettar, P. Narayankar and M. Patil, "An efficient method of detecting exudates in diabetic retinopathy: Using texture edge features," 2016 International Conference on Advances in Computing, Communications and Informatics (ICACCI), Jaipur, 2016, pp. 1188-1191, doi: 10.1109/ICACCI.2016.7732206.

[6] Imadjust, Mathworks Inc. [Online]. Available: https://in.mathworks.com/help/images/ref/imadjust.html, Accessed on 20th June, 2020

[7] Types of Morphological Operations, Mathwprks Inc. [Online]. Available: https://in.mathworks.com/help/images/morphologicaldilation-and-erosion.html, Accessed on 20th June, 2020

[8] Dilation and Erosion, Image Processing Toolbox User's Guide, Mathwprks Inc. [Online]. Available: http://matlab.izmiran.ru/help/toolbox/images/morph2.html,

Accessed on 20th June, 2020 